\title{
Hans Blumenberg y el feminismo
}

\section{Hans Blumenberg and Feminism}

\author{
Josefa Ros Velasco \\ Universidad Complutense de Madrid
}

Recibido: 15-12-2014

Aceptado: 11-09-2015

\section{Resumen}

El filósofo alemán Hans Blumenberg [1920-1996] se interesó por las cuestiones feministas durante las últimas dos décadas de su vida como muestran su dedicación y esfuerzo en la compilación de recortes seleccionados en los medios de comunicación relacionados con la temática de la mujer y su elaboración de múltiples ensayos cortos inspirados en estos últimos y en un sinfín de curiosas anécdotas. Hans Blumenberg y el feminismo pretende poner al lector en conocimiento sobre la existencia de una línea de investigación del pensamiento blumenberguiano totalmente inexplorada hasta el momento. La misma únicamente puede ser revelada a través del estudio del 'Nachlaß' del filósofo que se encuentra en el Deutsches Literaturarchiv de Marbach (Stuttgart) y que personalmente he tenido oportunidad de indagar gracias a la concesión de una beca de investigación del DAAD (Deutscher Akademischer Austage Dienst).

Palabras clave: Evolución, Feminismo, Hans Blumenberg, Hominización, Mujer, Nachlaß.

\begin{abstract}
The German philosopher Hans Blumenberg [1920-1996] was interested in feminist issues during the last two decades of his life as shown their dedication and effort in compiling selected cuts in the media related to the topic of women and their development of multiple short essays inspired by the latter and countless curious anecdotes. Hans Blumenberg and feminism seeks to inform the reader about the existence of a blumenberguian thought line of research totally unexplored so far. It can only be revealed through the study of the philosopher 'Nachla $\beta$ ' found in the
\end{abstract}


Deutsches Literaturarchiv Marbach (Stuttgart) which I personally had a chance to dig through the award of a research grant from the DAAD (Deutscher Akademischer Dienst Austage).

Keywords: Evolution, Feminism, Hans Blumenberg, Hominization, Woman, Nachla $\beta$.

\section{Efímeras pinceladas sobre la figura femenina en la obra primera y póstuma}

El erudito filósofo alemán Hans Blumenberg, ${ }^{1}$ conocido en la cultura occidental en un primer momento por sus trabajos hermenéuticos sobre el mito, la metáfora y lo inconceptualizable ${ }^{2}$ y en último término por sus esfuerzos en la tarea de conjugar una antropología fenomenológica, ${ }^{3}$ dedicó algunas líneas, en la práctica desconocidas, a la cuestión femenina. En el pensamiento blumenberguiano no podemos encontrar la confabulación de una teoría feminista contemporánea ni una reflexión constitutiva sobre la misma, pero sí aislados excursos a los que merece la pena prestar atención debido a su anonimato y su interés para la Filosofía contemporánea. Hasta el momento, ningún estudioso ha emprendido la terea de rescatarlos del lugar en el que descansan, hacerlos públicos y accesibles al resto de investigadores sobre el trabajo blumenberguiano y tratar de conectarlos entre sí para averiguar cuál era el interés que subyacía a la inquietud del filósofo por la mujer. Siguiendo lo anterior, el objetivo primordial de este artículo será poner a los lectores y estudiosos de la filosofía de Blumenberg en conocimiento de la existencia de tales documentos actualmente ignorados, abriendo las puertas a una posible investigación posterior que coloque esta pieza del puzle blumenberguiano en el lugar que le corresponda.

Al lector experimentado en el trabajo blumenberguiano editado hasta el presente, recogido tanto en su obra primera como en la póstuma, quizá le sorprenderá la

\footnotetext{
${ }^{1}$ Hans Blumenberg nació en Lübeck, el 13 de julio de 1920 y murió en Altenberge (Münster) el 28 de marzo de 1996. Estudió Filosofía en Paderborn y en Frankfurt am Main entre 1939 y 1941, finalizando sus estudios en Filosofía, Germanística y Filología clásica en la Universidad de Hamburgo tras la II Guerra Mundial. En 1950 obtuvo la habilitación a la Cátedra en Filosofía en la Universidad de Kiel. Asimismo fue profesor en las universidades de Giessen (desde 1960), Bochum (desde 1965) y Münster (desde 1970), donde consiguió ser profesor emérito en 1985. Consulta de la bibliografía completa en la web del Deutsches Literaturarchiv Marbach: www.dla-marbach.de

2 Selección de obras de referencia: Paradigmen zu einer Metaphorologie [Paradigmas para una metaforología], 1960; Die Legitimität der Neuzeit [La legitimidad de la modernidad], 1966; Arbeit am Mythos [Trabajo sobre el mito], 1979.

3 Selección de obras de referencia: Lebenszeit und Weltzeit [Tiempo de la vida y tiempo del mundo], 1986; Höhlenausgänge [Salidas de caverna], 1989; Beschreibung des Menschen [Descripción del ser humano], 2006.
} 
dedicación del filósofo al estudio del feminismo. Apenas si podemos encontrar referencias incomunicadas en la bibliografía de la que disponemos a la situación de la mujer. Como frágil ejemplo de una anotación de este cariz en un escrito que fue publicado en vida podemos aludir a la obra Das Lachen der Thrakerin. Eine Urgeschichte der Theorie [La risa de la muchacha tracia. Una protohistoria de la teoria], publicada por la editorial Suhrkamp en 1987. Este texto gira en torno a la historia que recoge el Teeteto de Platón, según la cual Tales de Mileto caminaba tranquilamente observando la grandeza de los astros cuando, repentinamente, cayó en un pozo. Una esclava tracia, que había observado la escena, estalló en carcajadas burlándose del sabio que quería conocer las cosas del cielo ignorando las que estaban junto a sus pies. Hacia el final del capítulo X, traducido en la edición castellana como "El cochero de Tico Brahe y el terremoto de Lisboa," Blumenberg narra la versión de la leyenda de Tales de Mileto del escritor inglés Samuel Richardson [1689-1761], como prueba de que la anécdota de Tales varía en cuanto a su desarrollo y sus personajes de forma constante a lo largo de la historia. En aquella, un hombre cae en un foso por andar mirando las estrellas y un pasante le recrimina por no ocuparse de los asuntos propios y dejar a un lado los de la imaginación que son más propios de los niños y las mujeres que admiten "cualquier impresión." (Blumenberg, 2009, 149-150). ${ }^{4}$ Es este pasaje Blumenberg no hace realmente ninguna reflexión a propósito de la asociación de la mujer a negocios menores que no pueden ser tomados en serio; pero merece la pena traer a colación la ausencia de la misma como hilo introductorio a lo que posteriormente desvelaremos.

Asimismo, como muestra - menos efímera que la anterior pero a su vez menos recurrida- de un tratamiento de la problemática de la mujer en una obra póstuma, podemos aludir al título Quellen, Ströme, Eisberge [Fuentes, corrientes, icebergs], publicado por Suhrkamp en 2012.5 La tercera y última sección de esta obra, elaborada entre los meses de septiembre a noviembre de 1980 y conocida como "Eisberge," indaga en la historia una metáfora que aunque ya estaba presente en la

\footnotetext{
4 "Una última observación sobre el siglo XVIII demuestra que la fábula esópica del astrónomo caído ha entrado en la pedagogía a través del divulgado compendio de Samuel Richardson Moral para la juventud, traducido al alemán por Lessing (...) El héroe de la historia no es más que un cierto observador de las estrellas que en plena dedicación a su oficio tropieza y cae en una fosa bastante profunda e intenta volver a salir de ella. Un hombre no insensato que pasaba por allí le amonesta con una combinación de casi todas las variantes usuales de la tradición: saca el provecho pertinente de este accidente; deja en adelante que las estrellas sigan tranquilamente su curso y preocúpate algo más de los fosos; pues ¿no sería insensato que por las estrellas predijeras a otra gente sus incidentes sin ver en ellas los tuyos propios? (...) A esa enseñanza viene añadida una "consideración" que se ocupa de la adivinación y de su tipología: la adivinación sería una de las trampas más peligrosas en la vida humana. Sobre todo para la credulidad de mujeres y niños, entre los que la imaginación es tan fuerte que, al ejemplo de la cera blanda, admite cualquier impresión" (Blumenberg, 2009, 149-150).

5 Véase la reseña en castellano: Ros Velasco, Josefa, "Quellen, Ströme, Eisberge», Res Publica (Madrid), n 29, 2013, pp. 127-131.
} 
Antigüedad no alcanzó su 'spitze' [cima] hasta los años veinte, cuando el hundimiento del Titanic despertó la atención de los curiosos por el fenómeno natural que es el iceberg. En un momento dado, encontramos a Blumenberg llevando a cabo un análisis de la aplicación de la metáfora del iceberg al problema de la violencia de género, denotando que, al igual que tras la insignificante 'punta del iceberg' se encuentra toda la arquitectura que la sostiene, más allá del delincuente que maltrata a una mujer podemos vislumbrar toda una construcción de lo que representa el fenómeno femenino y su miseria. En este contexto, presta especial atención a la violencia dentro del matrimonio y la dificultad que entraña descubrir la misma asemejándola al hecho de que, al igual que sucede con un iceberg, cuando la base del mismo desaparece, también acaba haciéndolo la superficie ["die Spitze des Eisbergs werde erst dann verschwinden" (Blumenberg, 2012, 216-217)], ${ }^{6}$ y ya no podemos darnos cuenta en absoluto de lo que está sucediendo.

Indudablemente, estas pinceladas sobre la mujer habrán de ser tomadas en cuenta a la hora de reconstruir el pensamiento filosófico blumenberguiano a la luz de sus eminentes intereses feministas. No obstante cualquiera puede acceder a tan efímeras notas debido a su naturaleza divulgada. Por sí solas no llaman la atención más que desde la perspectiva de encontrar como pocas veces la crítica blumenberguiana a la sociedad contemporánea y a un Blumenberg más mundano que de costumbre sufriendo las consecuencias de una época. Sin embargo, en conjunción con los iné-

\footnotetext{
6 "Wenn ein Pfarrer die These ausdrücklich >wagt`, daß die verwahrlosten Stadtstreicher nur die Spitze eines Eisbergs darstellen, möchte er das der Wahrnehmung aller sich darbietende quantitativ unbedeutende Phänomen mit einem ungleich und doch noch vergleichbar großen Untergrund ausstatten. (FAZ 26. Mai 1979) Als im Mai 1978 in Köln ein Tribunal mit dem Titel »Gewalt gegen Frauen« abgehalten wurde, eröffnete sich für ein breiteres Publikum eine ganz neue Dimension schwer kriminalisierbarer Delikte. Der Bericht darüber gipfelt in der Feststellung: Was dort von betroffenen Frauen berichtet wurde, ist dennoch nur die Spitze des Eisbergs mit Namen sweibliches Elendr. (Die Zeit 19/1978) Die neue Tochterdisziplin der Kriminologie erschien gerade rechtzeitig auf dem Plan, um auch diesen Komplex aufzufangen un dan das Licht wenigstens der Öffentlichkeit eines internationalen Kongresses, des III. Weltkongress für Viktimologie in Münster, zu bringen. Dort berichtet von neueren Untersuchungen über Vergewaltigungen der Freiburger Gerichsmediziner Peter Volk, da $\beta$ nur wenige Triebtäter unter den Männern sind, die Frauen vergewaltigen, die meisten in orderntlichen Verhältnissen leben, verheiratet sind, die Opfer schon vorher kennen und schon lange vor der Tat dazu entschlossen sind, auch mit Gewalt ihr Ziel zu erreichen. Unter den Tätern seien auffallend viele mit einem ausgeprägt traditionellen Frauenbild. Sie hätten geglaubt, wie sie angeben, das Sich-Zieren der Frau sei letztlich nur vorgetäuscht und könne mit Gewalt gebrochen werden. Nach einer Allensbacher Umfrage hätten 18\% aller verheirateten Frauen schon einmal eine Vergewaltigung in der Ehe erlebt, die traditionell als erlaubt gelte, und folglich müsse die Vergewaltigung im Sinne des Strafrechts blo $\beta$ als Spitze eines Eisbergs aufgefa $\beta t$ werden. Hier kann die Metapher sogar erweitert werden: die Spitze des Eisbergs werde erst dann verschwinden, wenn seine Basis verschwunden ist, das Bild von der ständigen sexuellen Verfügbarkeit der Frau in der Ehe. (Die Zeit 40/1979 vom 28. September 1979: Ingrid Rieskamp, Die Frau als Freiwild) Man kann sich vorstellen, da $\beta$ das neue Fach Viktimologie ein geradezu ideales Feld für die Verwendung der Eisberg-Metaphorik bietet” (Blumenberg, 2012, 216-217).
} 
ditos que expondremos a continuación, y que son de un difícil acceso, permiten descubrir una dedicación más que puntual a la investigación sobre la mujer en el pensamiento del filósofo y dan rienda suelta a la elucubración acerca del modo en el que la misma encaja en su corpus filosófico.

\section{El 'collage' sobre la mujer en la obra inédita}

\subsection{Inéditos a la vista en el DLA}

La obra inédita del filósofo, el 'Nachla $\beta$ ' blumenberguiano, se encuentra en el Deutsche Literaturarchiv (DLA), ubicado en el pueblo Marbach am Neckar (Stuttgart). Gran parte de su dedicación a la cuestión del feminismo se presenta a través de lo que se denominan 'kurz essays' [ensayos cortos] y 'kerteikarten' [tarjetas] y se localiza con facilidad entre los 'Handschriften' [manuscritos] del 'Katalog OPAC Kallías' del DLA Marbach al introducir en el buscador de texto libre ['Freitext'] la palabra alemana 'Feminismus' [feminismo] -no así la sinónima 'Frauenbewegung,' que no arroja por el momento resultado alguno-. Son ejemplos de ello el manuscrito de una página titulado Dem Feminismus zur Erinnerung [El feminismo en la memoria], del que desconocemos la fecha de escritura, y una carpeta destinada a la compilación de material variado ['Materialsammlung'] que ostenta el título Konvolut Materialsammlung Feminismus / Anthropologie [Colección de material variado de feminismo / antropología], cuya fecha de elaboración oscila entre los años 1988 y 1990, apenas unos años antes de la muerte del autor.

El primero de los documentos, Dem Feminismus zur Erinnerung, recupera una figura que es común entre los textos blumenberguianos: la de Adolf Hitler. Localizado en la carpeta ['Mappe'] 4 del estuche ['Schuber'] número 6 del 'Nachla $\beta$ ' y bajo las siglas UNF7 y localizador 2526, el inédito introduce la idea de que la mujer ha tenido a lo largo de la historia mundial el título de incomprendida y sufriente por una fama no reconocida que la ha relegado a la inferioridad. Sin embargo, destaca Blumenberg, hasta entre los más débiles existe la incorrección. Narra así la anécdota de que cuando Hitler fracasó en su intento de golpe de estado en Munich en 1923, y tras caer en una profunda depresión, fueron las mujeres quienes lo animaron y enderezaron. Hitler supo encontrar las palabras de gratitud oportunas y en una ocasión, a propósito del ochenta cumpleaños de una de estas mujeres, se presentó el 13 de julio de 1937 en Munich para honrarla. Para entonces,

\footnotetext{
${ }^{7}$ Existe una cierta polémica entre los estudiosos de la obra de Blumenberg acerca del significado de las siglas UNF, que bien podrían refereir a 'Unerlaubte Fragmenten' [fragmentos no autorizados] o a 'Unfertig' [inacabado].
} 
Hitler ya era jefe del 'imperio alemán' y ya no tenía necesidad alguna de estar del lado de los oprimidos, pero gustó de hacer honor a quien había sido un viejo miembro del partido. ${ }^{8}$ Mediante este breve escrito, Blumenberg parece estar aportando su 'granito de arena' ante la constante disputa acerca de la postura hitleriana frente a la figura femenina para tratar de esclarecer, apuntando como siempre una historieta ilustrativa, que el 'Führer' mantenía un cierto respeto y gratitud hacia las mujeres. Las ideas de Hitler acerca del sexo opuesto están expuestas abiertamente en el póstumo Monologe im Führerhauptquartier 1941-1944, elaborado por el historiador alemán Werner Jochmann [1921-1994] en 1980 a partir de las grabaciones del abogado Heinrich Heim [1990-1998] y que la editorial Sonderauflage publicó en el año 2000 (edición póstuma en castellano en Crítica, año 2004, bajo la comisión del historiador británico Hugh Trevor-Roper [1914-2003], titulada Las conversaciones privadas de Hitler, 1941-1944). Una lectura de las mismas puede, según la ocasión, fomentar tanto la imagen de un Hitler misógino, como la del canciller que se manifiesta a favor de la emancipación de la mujer y en contra de la violencia de género. Constantemente declara ideas tales como que la mujer necesita de la protección del hombre, que la mujer tiende a estar pensando todo el tiempo en el hombre o que la inteligencia no es en la mujer cosa esencial. Sin embargo, sostiene, todo ello no da derecho al hombre a someter a la mujer a torturas morales o materiales. Como a lo largo de los muchísimos textos publicados e inéditos que podemos consultar, 9 Blumenberg sigue tratando una vez más de descubrir la personalidad de quien le

8 "Zu den verkannten Ruhmestiteln der Frau in der Weltgeschichte soll es gehören, dass sie sich auf die Seite der Unterlegenen stellte. Doch auch unter den Schwachen gibt es die Falschen. Als Hitler 1923 mit seinem Putsch in München gescheitert war, geriert er in eine tiefe Depression. Es waren Frauen, die ihn aufrichteten und ermutigten. Hitler hat worte und Gesten des Dankes gefunden. Am 13.Juli 1937 verliess er eigens seinen Berchtesgadener Sitz und fuhr nach München, um eine dieser Frauen zu ihrem 80.Geburtstag auszuzeichnen. Da war er schon der Führer des Reiches und hatte keine Bedarf mehr für die Naturgabe der Frau, sich auf die Seite der Unterlegenen zu stellen. Die Achtzigjährige wurde denn auch dafür geehrt, dass sie Altparteigenossin war. Hellsicht fürs Künftige hatte sie gehabt, Intuition für den Kommenden - eine der anderen Adelsgaben ihren Geschlechts." Dem Feminismus zur Erinnerung (DLA Marbach).

9 Véase el capítulo 2 "La congruencia entre tiempo de la vida y tiempo del mundo como locura" ["Die Kongruenz von Lebenszeit und Weltzeit als Wahn"], sección segunda "La abertura de las tijeras temporales" ["Offnüng der Zeitschere"] de la obra Tiempo de la vida y tiempo del mundo [Lebenszeit und Weltzeit] y los inéditos del DLA Marbach (breve selección): Kein Bankkonto: Damagogie der Freiheit vom Geld; Ein Lebensthema; Konsequenz der Staatlichen Theorie des Geldes; Das KZ garantiert den Geldwert; Die Bergpredigt nach Hitler; Glaubte Hitler an die Sterne?; Zur ermordung Hitlers geboren - oder besser nicht geboren zu sein?; Politik: Schicksal, Kunst, Wissenschaft; Wer oder was man hätte wollen; Der Mann, der Hitler erschoss; Hitlers Bakterien; Die Absolute Unfreiheit des Artztes; Es wird werden, wie wenn nichts gewesen wäre; Familienväter; Definitionsversuch von Radikalismus: Abhängig von der Zweck/Mittel/Struktur. Para un análisis en mayor profundidad, consúltese el documento Kurzessays en el DLA Marbach, en el que aparece un índice que ocupa varias tarjetas rosas ['Karteikarten'] de los textos que tratan sobre Hitler bajo el título 'Adolfiana'. 
relegó durante un tiempo a la existencia en un campo de concentración por su condición de 'Halbjude' [medio judío], no dejándose embaucar por las apariencias de una falsa lucha por los derechos de la mujer.

Por su parte, la carpeta Feminismus representa una de las grandes pruebas de que Blumenberg se interesó por los movimientos feministas. En ella el filósofo ha guardado un sinfín de recortes de artículos, noticias, imágenes, entrevistas, etc., extraídos de revistas de antropología y cultura, periódicos diarios -entre los que destaca el FAZ (Frankfurter Allgemeine Zeitung)- y suplementos, seleccionados entre los años 1988 y 1990 -coincidiendo con la época en que Blumenberg se retiró de la vida de profesor y dejó de publicar obras-, en los que el tema de la mujer y el feminismo son los protagonistas.

\subsection{Inéditos escondidos en el DLA}

Existen, además de los citados, muchos otros documentos que se encuentran ávidamente escondidos bajo títulos que no auguran la temática en cuestión -algo que es frecuente en Blumenberg-y que evidencian un interés constante del filósofo por el tema que tenemos entre manos. Ello imposibilita, por otra parte, que la exposición del trato blumenberguiano de la cuestión feminista sea totalmente exhaustiva puesto que el hecho mencionado hace más que probable que existan escritos sobre el feminismo en el 'Nachla $\beta$ ' ocultos bajo títulos desconcertantes a los que personalmente no he accedido. A diferencia de lo que ocurre con otros bloques temáticos, como por ejemplo el que refiere a la cuestión 'Adolfiana,' citada en la nota al pie de la página anterior, Blumenberg no elaboró un índice de sus títulos sobre el tratamiento de la mujer. Sin embargo, este análisis no deja de merecer la pena en tanto que no sólo advierte de una nueva línea de investigación del pensamiento blumenberguiano totalmente desconocida sino que además nos permitirá conocer un poco más la filosofía de un autor para la que toda pista o indicación añadida es poca.

A medida que nos adentramos en la lectura de los manuscritos y las tarjetas sobre la mujer, podemos comprobar que no nos encontramos ante la confección de una teoría propiamente dicha sobre la filogenia u ontogenia femenina ni sobre el feminismo. Tampoco podemos calificar de reflexiones, con todo lo que ello conlleva, al conjunto de material que tenemos a nuestra disposición. Algunos de estos textos tratan sencillamente sobre la condición femenina, su esencia y naturaleza, sobre los movimientos feministas, acerca de anécdotas relacionadas con la temática o sobre la exposición del punto de vista de otros colegas sólo de manera frugal. Encontraremos, pues, a un Blumenberg que cuestiona las condiciones históricas del fenómeno femenino posicionandose, como en otras ocasiones, contra todo tipo de absolutismo: tanto si aquel consiste en defraudar a la mujer como si trata de exal- 
tarla en demasía. Comenzaremos, siguiendo lo anterior, rastreando los documentos a partir de los que Blumenberg inicia la búsqueda de la naturaleza biológica y cultural de la mujer para culminar con el escrutinio del camino que ha conducido a los absolutismos feministas.

En los albores de la cultura humana la función biológica prevalecía sobre las cuestiones emocionales, mentales o económicas. Los primates machos requerían aproximadamente el doble de tiempo que las hembras para convertirse en adultos, esto es, para hacerse cargo de enseñar a la descendencia, y eran las hembras primordialmente las encargadas de la crianza y la educación de la prole. Inspirándose en las palabras del antropólogo canadiense Lionel Tiger y el antropólogo inglés Robin Fox, que aparecen en su obra conjunta The Imperial Animal (1971), Blumenberg reconoce que "hay una clara diferencia entre el proceso de aprendizaje masculino y femenino. Las estructuras de aprendizaje del macho son más complejas y exigentes, los de la mujer tienen lugar en un espacio más limitado."10 Esta pudo ser una de las causas principales por las que en las sociedades cazadoras-recolectoras del Mesolítico los hombres unidos por la caza y exentos durante más tiempo de las tareas instructivas comenzaron a intercambiar a sus mujeres para aprovechar su madurez sexual. Incluso las rivalidades entre clanes quedarían a un lado a la hora de establecer vínculos carnales en tanto que nadie podía permitirse el lujo de eliminar a quien le proveía con mujeres. ${ }^{11}$ Así, a diferencia de las poblaciones animales, las humanas sustituyeron su ecología por una economía constituida sobre el acto de compartir a las mujeres. En adelante, los núcleos familiares entregarían a las jóvenes hijas a cambio de una parte de las propiedades del contrayente, dando lugar a la cosificación de la mujer que, como si de un "cheque en blanco" ["Bankoscheck"] se tratase, encarnaba "la forma más primitiva de propiedad" ["die urtümlichste Form des Eigentums"] y el derecho del hombre sobre la misma.12

10 "Die Lernzeiten sind nicht nur artspezifisch, sondern auch geschlechtsspezifisch. Die männlichen Primaten benötigen etwa doppelt so viel Zeit wie die weiblichen, um ihre Rolle als erwachsene Tiere - und das heisst im Prinzip: solche, die auch wiederum lehren könnten - zu übernehmen. (Tiger-Fox, 206) "Es besteht ein eindeutiger Unterschied zwischen dem männlichen und dem weiblich Lernablauf. Die Lernstrukturen des Männchens sind komplexer und anspruchsvoller, die des Weibchens räumlich begrenzter." (a.a.O. 208). Säugetiere sind Lerntiere, der Mensch ist dazu noch ein Lehrtier (DLA Marbach).

11 "In der steinzeitlichen Jägergesellschaft holen sich die Männer einer durch die Jagdziele verbundenen Gruppe ihre Frauen aus einer entsprechenden anderen oder mehreren anderen Gruppen und treten dafür die Frauen aus der eigenen Gruppe ab. Dadurch wird zwischen den Gruppen in bezug auf Frauen und damit in bezug auf Nachkommen Abhängigkeit hergestellt, die auch dann nicht zerstört wird, wenn diese Gruppen, etwa durch Rivalität bei der Jagd und Jagdbeute, feindlich aufeinandertreffen. Die biologische Funktion überlagert die emotionale, ökonomische und psychische Funktion. "Niemand kann es sich leisten, die Leute auszulöschen, die ihn mit Frauen versorgen." Exogamia als erste Institution (DLA Marbach).

12 "Eine andere Formel für diesen Sachverhalt lautet: Menschen haben einen Sinn für 'Eigentumlich' (Tiger-Fox, 161) Primaten teilen nicht und tauschen nicht; das Teilen auf Gegenseitigkeit ist vielleicht 
Trás siglos de perpetuación de un sistema patriarcal y cuando los problemas biológicos quedaban en un segundo plano debido a la supresión de la economía feudal y al fomento de la industrialización, la mujer seguía siendo un medio para el hombre en un sentido bien distinto. La abundancia de la modernidad democratizó el aburrimiento y esta se convirtió en el reclamo de entretenimiento de los hombres. Un ejemplo de ello es recogido en la tarjeta Unbehagen XLV: Langeweile + Aktionismus [Malestar XLV: Aburrimiento + Accionismo], en la que Blumenberg recordará que el dramaturgo austríaco Arthur Schnitzler [1862-1931], quien padecía de un aburrimiento inquebrantable, recurría a las féminas para salvarse de aquel. 13

Siguiendo a Blumenberg, "el pasado de la mujer fue la clave indudable para su futuro" ["die Vergangenheit der Frau der Schlüssel zu ihrer Zukunft ist"]. Hasta entonces el silencio de las mujeres era su ocultamiento forzado de aquellos verdaderos deseos a los que no podían escapar. La materialización de los mismos representó un gran potencial para los levantamientos de los años veinte. El deseo, el autoconcepto o la identidad aproximada de la conducta y la conciencia, sostiene Blumenberg, fueron aspectos definitivos en un levantamiento que determinaría lo que se avecinaría para la mujer. La posteridad demandaría "un relieve mayor de la mujer" ["ein noch schärfer geprägter Typus von Frau"] que, en pleno proceso de liberación -nótese, por ejemplo, el comienzo del uso de "los anticonceptivos" ["die Kontrazeptiva"]-, se sublevaba del hombre y abría las puertas a la selección de los

die früheste Form des Tausches, insbesondere auf einer Stufe, auf der die Arbeitsteilung so vollzogen ist, dass die Frauen Vegetabilien sammeln, die Männer Fleischbeute jagen. Auf diese Weise wurde ein Formular erlernt, das auch auf andere Beziehungen angewendet werden konnte: Tausch der Frauen, Tausch der heiratsfähigen Totcher gegen einen Anteil an der Beute. 'Der Durchbruch zum Menschen brachte die Evolution der Vorstellung mit sich, dass die Frau ein Eigentum sei - vielleicht die urtümlichste Form des Eigentums. Die erste Schuld der Welt war vermutlich eine Frau, der erste Blankoscheck höchstwahrscheinlich das Recht eines Mannes auf seine - geborene oder ungeborene Totcher, das er einem anderen Mann abtrat' (Tiger-Fox, 163) (...) Der Kern unserer Argumentation ist der, dass der Austausch ein fundamentaler menschlicher Akt ist und dass seine Verweigerung eine Verweigerung der Menschlichkeit gleichkommt." (a.a.O. 197) Alle Tierpopulationen haben Ökologien, nur die menschlichen Populationen haben Ökonomie (DLA Marbach).

13 "Wagner, Schnitzler 30: Was ihn am meisten stört: die Langeweile. Fast immer, wenn er sich zu Bett legt, empfindet er eine gewisse Angst vor der Leere und Gleichförmigkeit des folgenden Tages. Die beiden Feststellungen sind schwer vereinbar: einerseits Sturm und Drang, anderseits Langeweile. Die erotische Aktivität des von Schnitzler dramatisch konzipierten Typs ist von der Langeweile oder besser: von der Furcht vor der Langeweile angetrieben (...) Deshalb ist auch die FEststellung der Biographie nicht zutreffend, die ständige und leichte Befriedigung seines Bedürfnisses nach Partnerinnen habe ihn schliesslich vor der Langeweile gesichert: 'Jedenfalls geht es auf das Konte der Frauen, dass der junge Mann, der sich vor nicht allzu langer Zeit noch tödlich gelangweilt hat, im Februar 1883 in s Tgb notiert, die Zeit gehe hin \& man wisse nicht, wie' (a.a.O.35). Unbehagen XLV: Langeweile + Aktionismus (DLA Marbach). [Los errores ortográficos y la aplicación del principio de economía al lenguaje han sido transcritos según aparecen en el manuscrito original sin alteraciones por parte de la autora del artículo.] 
sexos. ${ }^{14}$ Sin embargo, Blumenberg advierte que quizá el augurado futuro emancipador de la mujer se vio exponencialmente desvirtuado por el surgimiento de las posturas feministas más radicales.

El feminismo de la diferencia que abogaba por posturas tan extremas como la defensa de la partogénesis o del levantamiento contra los derechos paternales de los progenitores masculinos, por recoger dos de los ejemplos sobre los que Blumenberg se detiene en mayor profundidad, llegaron a insertar en el movimiento un cierto toque de burla. Por un lado, una idea tal como la partogénesis, que busca la posibilidad de la reproducción sin necesidad de fecundación por medio del desarrollo de células femeninas, dejaba de lado al sexo masculino y apostaba por lo que el filósofo ha denominado la creación de 'parques zoológicos' artificiales: "Parthogenese bei homo sapiens sapiens hinterlässt nur ein etwas müdes museales Interesse an der

14 "Die Herzzerreissenden Schilderungen, die im Zuge des sog. Feminismus von der Vergangenheit der Frau gegeben worden sind, sollten auf eine Zukunft von Befreiungen als blossen Negationen jener Vergangenheit vorbereiten und einstimmen. Der Grundgedanke ist zweifellos richtig, dass die Vergangenheit der Frau der Schlüssel zu ihrer Zukunft ist. Der elementare Sachverhalt dieser Vergangenheit war, dass die Frau sexuelle Verbindungen eingehen musste, um ihre Existenz zu sichern oder zu verbessern, folglich ohne Rücksicht auf ihr Verhältnis zu ihrer Sexualität Mutter wurde und Nachkommenschaft aufzog. Aber: Auch nur sie konnte es. Denn im Unterschied zum Mann ist der Anteil der Frau am Sexualakt, ihr wirklicher Lustgewinn oder ihre reelle Abneigung, nicht nachprüfbar, so viel sich auch die männlichen Partner sorgen mochten, ob sie diesen Erfolg wirklich erzielten. Die Frau repräsentierte ein undurschsichtiges Sexualwesen. Nur in sehr gesicherter Position konnte sie ihre Frigidität oder sogar ihre gleichgeschlechtliche Neigung zu erkennen geben und die klassische 'Rolle' verweigern. Genetisch betrachtet, zwingt dieser Sachverhalt zu der Hypothese, dass alle sexuellen Abweichungen von der proliferativen Funktion durch indifferent bis widerwillige Mutterschaften für beide Geschlechter übertragen wurden. Dass der Anteil solcher Abweichungen beim Menschen relativ gro $\beta$ ist, muss gerade an der versteckten Mitwirkung der Frau bei diesem Erbfaktor gelegen haben. Es ist klar, dass bei dem Widerstand der Frauen gegen die Fortsetzung dieser Geschlechtgeschichte diejenigen führend waren, die bis dahin ihrer schweigendwiderwilligen Verhehlung ihrer wahren Wünsche nicht hatten entgehen können. Sie bildeten ein starkes Potential des Aufstands und er für die modische Bereitwilligkeit der anderen perzeptiven Ausformung eines neuartigen Typs, dessen Reize schon in den zwanziger Jahren vorgeführt und imitiert worden waren. Dies Ganze war nur möglich, weil es nicht mehr um die Existenz ging. Aber nun ging es auch nicht mehr um den Anteil am Genom: Dieser Typus wird sehr schnell verschwinden und überlagert werden durch den Vererbungsanteil derjenigen Frauen, die heterosexuelle Verbindungen aus Neigung, Lust, Trieb, Selbstauffassung - jedenfalls in der annähernden Identität con Verhalten und Bewusstsein, Ausdruck und Disposition - eingegangen sind. Sie bestimmen, was kommt. Dieser unausbleibliche Umschlag muss genetisch genau die Folgen haben, die der ganzen bis dahin eingetretenen Entwicklung zuwider laufen: Ausschaltung sexueller Indifferenz oder Abweichung, Verstärkung der sexuellen Prägungen bei beiden Geschlechtern. Nur als Verstärkereffekt muss in den Kalkül einbezogen werden, dass die Kontrazeptiva nicht einmal mehr den primären Sexualerfolg des Lustgewinns massgebend machen: Nachkommenschaft wollen wird ein noch schärfer geprägter Typus von Frau als der fortan schon sein muss, der überhaupt den Sexualakt wünscht. Die Selektion programmiert eine unerwartete Naturmässigkeit der Geschlechter." Die Zukunft der Frau (DLA Marbach). 
maskulinen Seite."15 Por otro lado, denuncia que la propuesta del feminismo matriarcal que pretende derrocar la legitimidad biológica de los padres a conocer a sus hijos condena a los hombres a una invertidumbre insoportable que roza la "violación de un derecho humano" ["Verweigerung eines Menschenrechts"]. ${ }^{16}$

Puede que tal intensidad sea el motivo por el cual algunas de las mayores influencias del filósofo se han mofado tanto de la mujer como del feminismo. Sin ir más lejos, Blumenberg recoge en términos neutrales en la tarjeta Können Frauen philosophieren? [¿Pueden las mujeres filosofar?] (DLA Marbach) el testimonio de la tesis del escritor alemán Thomas Mann [1875-1955] acerca de la incapacidad de las mujeres para la labor filosófica. Reflexionando sobre la obra de la escritora alemana Gabriele Reuter [1859-1941], Aus guter Familie [De buena familia], editada por Fischer en 1895, Mann se posicionó como un antifeminista alzado contra las confesiones y el autodescrubrimiento de las novelas femeninas de la época. Blumenberg recuerda en su texto cuando Mann visitó Berlin en el año 1903 y mantuvo una conversación con Gabriele Reuter acerca de su actitud ante los derechos de la mujer, a partir de la que escribió un artículo periodístico en el año siguiente. Las palabras de Mann eran claras:

Una vez asistí a una reunión de una asociación de mujeres en Munich, le doy mi palabra. En el orden del día aparecía la pregunta: ¿Pueden las mujeres filosofar? Un profesor universitario intervino y el resultado fue la afirmación triunfal acerca de que las mujeres sí podían filosofar con un fin claro y apasionado como los hombres. Ahora le

15 "Dabei kann noch die Vererbung viel rationeller betrieben werden, als jetzt, wo sie so vielen Zufälligkeiten unterworfen ist. Parthogenese bei homo sapiens sapiens hinterlässt nur ein etwas müdes museales Interesse an der maskulinen Seite: Es werden davon nur wenige Exemplare für die zoologischen Gärten erzeugt. Mit der Befriedung der Geschlechter und der Lösung aller mit ihrer Differenz verbundenen, gerade eben in Wien voll erschlossenen Probleme würde nicht nur Mann als Kuriosität der Schaustellung funktionloss. Wurde nicht einmal dem Zootier 'Menschenmann' sein Weintrost vom Zoofeminat gelassen?" Refugium im Zoo (DLA Marbach).

16 "Darf die Weigerung einer Frau, den Vater ihres Kindes aktenkundig zu machen - ein Grenzwert feministischer Exekution des Matriarchats - rechtspolitisch als Bagatelle der Nichtausübung des Versorgungsanspruchs behandelt werden? Opportunität beiseite - kein Politiker dürfte wagen, im Spätfeminismus seine Partei mit diesem Problem zu belasten. Doch wird man fragen müssen, wie ernst das Recht des Kindes genommen werden muss, seinen Vater zu kennen. Es ist ja dessen Erbe im biologischen wie im rechtlichen Sinne; im letzteren noch nicht lange den ehelichen Kindern gleichgestellt. Die Ungewissheit einem Menschen bewusst aufzuerlegen, wer denn sein Vater sei, um die Ausschliesslichkeit der Mutterrolle zu behalten, ist Verweigerung eines Menschenrechts. Zahllose sind auf die Suche nach ihren Vätern gegangen, sobald sie sich dessen bewusst wurden, dass deren Abwesenheit auf einem Bruch in ihrer Vor- und Frühgeschichte beruhen musste. Frauen kennen nichts Entsprechendes zu der Ungewissheit eines Mannes, ob er Vater ist - überhaupt oder im gegebenen Fall -; sie sollten nicht die ideologisch gezüchtete Frivolität zugebilligt erhalten, nach Zeitgeistlage eine weitere lebensverändernde Ungewissheit in die Welt zu bringen: den Vorenthält der Vateridentität." Im Spätfeminismus (DLA Marbach). 
pregunto: ¿Qué sentido tiene filosofar si pueden hacerlo las mujeres? Entonces puede hacerlo todo el mundo, por lo tanto no debería hacerlo nadie. Estoy dispuesto a casarme con una mujer si le gusta lo contrario a la filosofía. Gabriel Reuter respondió con una sonrisa indulgente, recordando que los hombres son como los niños que se ponen de acuerdo para reunirse y alentarse. ${ }^{17}$

De la misma manera, el propio Blumenberg da cuenta de que debido a la causa absolutista de la doctrina feminista la emancipación de la mujer no fue tomada todo lo suficientemente en serio que merecía y acabó asociándose en el imaginario de principios del XX a absurdos como el consumo de tabaco o la moda. ${ }^{18}$ En última instancia, recurre a las anécdotas sobre el canibalismo entre los parientes homínidos para representar metaforicamente el extremismo femenino. En el original Von Samoa bis Gombe - Nur Enttäuschungen [Desde Samoa a Gombe - sólo decepciones] (DLA Marbach) va a contar el ejemplo de una madre y una hija que tratan de robar a un recién nacido de su grupo para comérselo, como donosa asemejanza de sus palabras. 19

17 "Thomas Mann über Gabriele Reuter, die mit einem frauenrechtlerischen Roman »Aus guter Familie« in den neunziger Jahren berühmt geworden war und die Thomas Mann 1903 in Berlin besuchte und über die er am 14. und 17. Februar 1904 einen Zeitungsaufsatz veröffentlichte, seine zweite publizistische Arbeit. Über sein Gespräch mit Gabriele Reuter in Berlin und über seine Stellung zur Frauenrechtsbewegung schreibt Thomas Mann: "Also gut!... Ich habe einmal in München einer Frauenversammlung beigewohnt, auf mein Wort, ich bin hingegangen. Auf der Tagesordnung stand die Frage: Können Frauen philosophieren? Es war ein wildbewegter Abend; sogar ein Universitätsprofessor griff ein, und das Ergebnis war die sieghafte Bejahung der Frage, ob Frauen philosophieren könne. Übringens war man ja zu diesem Ende zusammengekommen. Ein bleicher und leidenschaftlicher Herr, der sich aufstellte und das Resultat aus wissen Gründen anzweifeln zu müssen glaubte, ward niedergemacht; der Universitätprofessor streckte ihn zu Boden. Nun frage ich aber jedermann: was soll das Ganze! Wenn eine Frau sphilosophieren` kann, so möge sie sich doch hinsetzen und es tun! Niemand wird sie hindern, niemand ihr übelwollen. Ich bin bereit, sie zu heiraten, wenn sie mir sonst gefällt. Aber wozu sich zusammenrotten... Gabriele Reuter habe darauf mit nachsichtigem Lächeln geanwortet, er müsse bedenken, dass es gleichsam Kinder sind, die sich verabreden, sich Mut machen müssen...” Können Frauen philosophieren? (DLA Marbach).

18 "Über Jahrzehnte hinweg ist es der Zigarettenindustrie nicht gelungen, das gewaltie Potential der weiblichen Kunden $\mathrm{zu}$ erschliessen; erst als diese aus anderen Gründen nach Symbolen der Emanzipation suchten, wurde die Industrie erfolgreich mit ihrer Werbung bei dieser Hälfte der Bevölkerung, während die andere von ihr abzufallen begann auch deshalb, weil sie ein Statussymbol verloren zu haben glaubte. Berufe sind den Menschen noch nie langweilig geworden, bis zum dem Augenblick, in dem ihnen die Kontingenz der eigenen Berufswahl dadurch bewusst gemacht wurde, dass es staatliche Angebote der Umschulung und des Berufswechsels gab (sie kosteten den Steuerzahler 1975 fast drei Milliarden DM) (Gehlen WW VII 325)." Das Land der Langeweile (DLA Marbach).

19 "Tiere in ihrem artschonenden Anstand täten so etwas nicht. Dieser schöne Vorbildlichkeit ist selbst für unsere nächsten hominiden Verwandten zerstört. Jane Goodall hat bei ihren ebenso lieben wie wilden Schimpansen drastische Fälle von Kannibalismus nicht übersehen können. Sogar jedem Feminismus hohnsprechende: Ein Mutter-Tochter-Paar hatte sich darauf verlegt, die Neugeborenen 
Pero entre las desfavorecedoras notas sobre la mujer, hacia el final del texto Zwei Theoretiker des Geldes [Dos teóricos del dinero] (DLA Marbach), encontramos el caso contrario, esto es, el del sociólogo alemán Georg Simmel [1858-1918], quien se casó con una filósofa, Gertrud Kinel, o como se la conocía en sus obras firmadas con pseudónimo: Marie-Luise Encke. Ella trabajó en la memoria del aniversario del movimiento por las mujeres que se elaboró después de la aprición del suplemento dominical del National Zeitung del 27 de noviembre de 1892. Simmel tenía en mente el libro de Theodor Gottlieb von Hippel que era del siglo anterior y versaba acerca de la mejora cívica de las mujeres. Dos años más tarde, Simmel escribió en Vossische Zeitung del 21 al 28 de octubre de 984 el artículo "El militarismo y la posición de las mujeres en la tesis de Aristóteles". En este recordaba a Herbert Spencer en el contexto de la guerra y a las mujeres presas. Así, concluye Blumenberg, el fundador de la sociología alemana es un ejemplo temprano de la reflexión por la mejora de las condiciones de las mujeres en una línea equilibrada aunque su postura sea poco conocida. ${ }^{20}$

Hasta aquí llega una exposición del tratamiento blumenberguiano de la historia de la cuestión femenina a la que hemos tratado de conferir un sentido secuente en la medida de lo posible. Partiendo del análisis de una situación ancestral en la que el intercambio de mujeres resultó ser uno de los pilares fundadores de la sociedad económica que se prolongaría durante los siglos posteriores hasta llegar al punto en que las mismas decidieron tomar las riendas de sus destinos - no sin obviar que la metodología, como hemos observado, quizá no fue la más acertada en algunos casos-, el interés de Blumenberg por este sexo ha quedado manifiestamente señalado. Queda pendiente, no obstante, mostrar que Blumenberg ha hecho hincapié en

ihrer Gruppe zu rauben und zu fressen. Dabei hätten wir sie so gern bei friedlichem Vegetarismus gewusst." Von Samoa bis Gombe - Nur enttäuschungen (DLA Marbach)

20 "Simmel war mit einer Philosophin verheiratet, Gertrud Kinel, die für ihre Werke des Pseudonym Marie-Luise Enckendorf bevorzugte. Aber nicht sie war es, die zwei Jahre nach der Eheschließung in der Sonntagsbeilage der 'National-Zeitung' vom 27.November 1892 über 'Ein Jubiläum der Frauenbewegung' schreibt. Es war Georg Simmel, der damit des ein Jahrhundert zurückliegenden Erscheinens von Theodor Gottlieb von Hippels Buch 'Über die bürgerliche Verbesserung der Weiber' gedachte, den Autor in die Reihe Hamann, Herder, Lichtenberg, Hippel stellen und den Zeiten der Mosesblicke (auf Gelobte Länder wohl) einordnen. Wie den 'Weibern' verschlechtert worden war, was nun verbessert werden sollte, ist für den Begründer der deutschen Soziologie ein frühes Musterstück: Durch die Sesshaftwerdung der Jäger und Sammler wand das Weiss allmählich die Befehlshaberin der Hausthiere und ehe sie sich dessen versah das erste Hausthier selbst. Zwei Jahre später schreibt Simmel in der 'Vossischen Zeitung' vom 21. und 28. Oktober 1894 'Der Militarismus und die Stellung der Frauen' über des Aristoteles These, sehr kriegerische Völker überliessen in ihren häuslichen Umständen die Herrschaft den Frauen, wogegen Simmel mit Herbert Spencer an den Zusammenhang von Kriegertum und Frauenbeute erinnert. Diese beiden frühesten Belege müssen genügen, um noch einen Blick auf die beiden Geldtheoretiker in Strassburg zu werfen. Es ist unwahrscheinlich, dass Knapp von Simmels Stellung zur 'Frauenfrage' gewusst hat." Zwei Theoretiker des Geldes (DLA Marbach). 
el hecho de que el siglo pasado no representa la única ocasión en la que la mujer ha tratado de revertir su posición de sexo débil.

Más allá de aquella primera revisión, si realizamos un análisis en detenimiento, observaremos que Blumenberg confiere un papel filogenético y ontogenético a la mujer que resulta definitivo en la evolución humana y en su lucha no sólo por sobrevivir sino por supervivir, siguiendo la terminología empleada en su póstuma Beschreibung des Menschen (2006). ${ }^{21}$ El filósofo esbozó toda una tesis acerca de la inversión ontogenética de la filogenética superioridad de los fuertes frente a los débiles que podemos encontrar en algunos de sus manuscritos del 'Nachla $\beta$ ' y que representa "el problema básico de la historia humana" ["Das Verhältnis der Schwachen und der Starken ist das Grundproblem der Menschheitsgeschichte."] explica Blumenberg en el inédito Die Koalition der Schwachen und die Sklaverei der Eliten (DLA Marbach). Según apunta,

El hecho más sorprendente de la selección en el proceso de hominización es que no ha habido preferencia exclusiva por los privilegios físicos. (...) Así que la expresión de otras cualidades debe haber sido también una ventaja en la lucha por la existencia sexual de los débiles físicamente. Uno casi no puede evitar argumentar que los primates machos podrían haber permitido que esto sucediese. El elemento femenino también es de consideración particular en el caso de los débiles físicamente, han sido un individuo intelectualmente excepcional por una conducta que combina cuidado y sexualidad. La propia enfermedad, por ejemplo cuando se piensa en la tuberculosis sufrida por personas de gran capacidad intelectual, no interfiere en las posibilidades de procreación, puesto que es algo natural en la historia de los animales. ${ }^{22}$

Volviendo sobre una idea expresada en Alle Tierpopulationen haben Ökologien, nur die menschlichen Populationen haben Ökonomie (DLA Marbach), los

\footnotetext{
21 Traducción al castellano, Descripción del ser humano, FCE: Buenos Aires, 2011, 662.

22 "Die erstaunlichste Tatsache der Hominisierung ist die Selektion ohne ausschliessliche Bevorzugung der physischen Privilegien. Es muss doch berücksichtigt werden, dass intellektuelle und musische Fähigkeiten sehr oft nicht mit physischen Vorzügen verbunden sind, also die Chancen im Wettbewerb um Fortpflanzung nicht an diese Verbindung gekoppelt sein können, wenn überhaupt im Gebestand der Menschheit intellektuelle und musische Qualitäten erhalten bleiben sollten. Es muss also auch einen Vorzug der physischen Schwäche bei Ausprägung anderer Qualitäten im sexuellen Kampf ums Dasein gegeben haben. Man wird schwerlich behaupten wollen, dass die männlichen Primaten diesen Sachverhalt ermöglicht haben können. Es muss in der weiblichen Initiation ein spezifisches Element der Beachtung auch des körperlich schwachen, geistig hervorragenden Individuums gegeben haben, eine Art Verbindung von Pflegeverhalten und Sexualität. Selbst Kranke, wenn man etwa an die Rolle der Tuberkulose bei intellektuell hochrangigen Individuen denkt, hat die Chancen der Fortpflanzung nicht so beeinträchtigen können, wie es in der tierischen Vorgeschichte ganz selbstverständlich war." Weibliches Zuchtwahlverhalten und überleben d Schwachen als Kulturträger [El comportamiento de la selección femenina y la supervivencia del débil como portador de la cultura] (DLA Marbach).
} 
débiles, entre los que se incluían las mujeres, aprovecharon con astucia la ausencia cada vez mayor de los cazadores y su disposición en el grupo para obtener ventajas sobre los otros miembros del grupo. Se equivocó entonces quien planteó que el único ser humano de pleno derecho era quien se encargaba de proporcionar lo necesario para sobrevivir, pues planteaba una ley económica que probablemente nunca había existido. 23

Como sugiere el titular que encontramos en uno de sus recortes del Frankfurter Allgemeine Zeitung del 17 de mayo de 1978, la asignación de la caza a los hombres y de la recolección a las mujeres y niños no fue en detrimento de este último par, quizá más bien de los primeros, quienes quedaron encargados de hacer la guerra para la posteridad. ${ }^{24}$ Hablamos de una selección femenina de los machos excelen-

23 "Wer behauptet, der einzig vollwertige Mensch sei derjenige, der die gesamte zum Überleben notwendige Arbeit selber leistet (...)" Alle Tierpopulationen haben Ökologien, nur die menschlichen Populationen haben Ökonomie (DLA Marbach)

24 Blumenberg, siguiendo a los antropólogos Tiger y Fox, afirma que la guerra no es una cuestión humana, sino un problema masculino: sólo los hombres cazaban y sólo los hombres fueron juntos a la batalla: "Die erstaunlichste Tatsache der Hominisierung ist die Selektion ohne ausschliessliche Bevorzugung der physischen Privilegien. Es muss doch berücksichtigt werden, dass intellektuelle und musische Fähigkeiten sehr oft nicht mit physischen Vorzügen verbunden sind, also die Chancen im Wettbewerb um Fortpflanzung nicht an diese Verbindung gekoppelt sein können, wenn überhaupt im Gebestand der Menschheit intellektuelle und musische Qualitäten erhalten bleiben sollten. Es muss also auch einen Vorzug der physischen Schwäche bei Ausprägung anderer Qualitäten im sexuellen Kampf ums Dasein gegeben haben. Man wird schwerlich behaupten wollen, dass die männlichen Primaten diesen Sachverhalt ermöglicht haben können. Es muss in der weiblichen Initiation ein spezifisches Element der Beachtung auch des körperlich schwachen, geistig hervorrrangenden Individuums gegeben haben, eine Art Verbindung von Pflegeverhalten und Sexualität. Selbst Kranke, wenn man etwa an die Rolle der Tuberkulose bei intellektuell hochrangigen Individuen denkt, hat die Chancen der Fortpflanzung nicht so beeinträchtigen können, wie es in der tierischen Vorgeschichte ganz selbstverständlich war. Tiger-Fox, 238: Nur unter der Voraussetzung der Chancen auch für körperlich schwache Individuen konnte sich eine Vielfalt des Genbestandes herausbilden, in der intellektuelle und musische Qualitäten ihren Anteil haben. Wenn das si ist, muss die Grundthese falsch sein, die Bevorzugung der Schwachen durch Medizin und Sozialismus habe der natürlichen Auslese ein Ende bereitet, ihr sogar zuwidergehandelt. Nur ist die Frage, ob Medizin und Sozialismus in der Verallgemeinerung ihres Schutzverhaltens dieselbe selektive Leistung zu erbringen vermögen wie die sexuelle Beachtung des physisch schwachen, aber geistig hervorrangenden männlichen Individuums in der weiblichen Zuchtwahl. Man wird vielmehr annehmen müssen, dass das zulässige Mas san körperlicher Schwäche oder sogar Krankheit bei individueller Selektion im Schnitt abhängig war von der Kompensation durch die andere Seite der menschlichen Qualitäten. Das kann ein abstraktes oder technisches System nicht leisten, und insofern ist die Gleichsetzung dieser Funktionen mit denen der physisch moderierten Zuchtwahl, wie sie Tiger und Fox vornehmen, wohl doch nicht zulässig. Der menschheitsgeschichtliche Grund für diese Differenz ist in dem folgenden Satz andgegeben: Der Krieg ist keine menschliche, sondern eine männliche Aktivität; der Krieg ist kein menschliches, sondern ein männliches Problem. Nur die Männer herrschten, nur die Männer jagten, und aus dieser einseitigen Geschlechtsorientierung ergab sich eine weitere: Nur die Männer zogen gemeinsam in den Kampf (a.a.0. 277)." Weibliches Zuchtwahlverhalten und überleben d Schwachen als Kulturträger (DLA Marbach). 
tes que han compensado su debilidad física mediante el desarrollo de otras cualidades humanas:

Cada movimiento de peso que hacían las madres facilitaba en gran medida que los débiles alcanzasen la posibilidad de la supervivencia. Los cazadores y los guerreros salían, las madres con los niños y los débiles ocultos entre ellos se quedaban a proteger las cuevas y las chozas. Y apareció la gran compensación: los débiles, sin embargo, buscaron el afecto de los demás y comenzaron a cantar y a girar en torno a ellos haciendo aquello para lo que los otros no tenían ni tiempo ni ganas - convirtieron la vida en algo más valioso que la mera supervivencia. La protección de los débiles, llevada a cabo por las madres, resultó ser la esencia y el suelo de la cultura. El pensamiento del débil trajo una poderosa alegría, condujo el aburrimiento de las noches oscuras - convirtió al débil en fuerte. 25

El análisis del rol de la mujer en la antropogénesis 26 que demuestra que su papel no puede ser desdeñado respecto al del hombre y que fueron capaces de sobreponerse a los designios biológicos. La neotenia es un factor ligado a la función de la madre sin el cual nuestra história evolutiva habría sido muy distinta. Las madres se encargaban de cuidar de la descendencia hasta edades muy avanzadas lo que incurre en que:

Casi al final el débil todavía tiene la oportunidad impredecible de ser el más fuerte, sólo porque sus madres les habían impuesto lo totalmente antinatural para obtener el ausente derecho a la supervivencia. (...) Así los débiles habrían hecho de su debilidad la

25 "Jede Gewichtsverschiebung zu den Müttern erleichterte das Los der Schwachen, hob ihre Überlebenschance. Die Jäger und Krieger zogen aus, die Mütter mit den Kleinen, unter ihnen verborgen die Schwächlichen, blieben im Schutz der Höhlen und Hütten. Und da entstand die grosse Kompensation: die Schwachen suchten dennoch die Zuneigung der anderen, sie fingen an zu singen und zu spinnen, sie dachten aus, wozu die anderen nicht Zeit und Neigung hatten - sie wurden die die das Leben neben dem Überleben noch lohnend machten. Der Schutz der Schwachen, von den Müttern erfunden, wurde zum Wesen und Boden jede Kultur. Die Schwachen dachten sich aus, was den Starken Freude brachte, die Langeweile der dunklen Nächte vertrieb - aber sie dachten sich auch aus, was die Schwachen stark machen würde." Die Koalition der Schwachen und die Sklaverei der Eliten (DLA Marbach).

26 "Die Rolle der Frau in der Anthropogenese: Neotenie \& Fesselung an die Mutterrolle sind Faktoren, ohne die es die A. A. nicht gegeben hätte. Erst wenn der Staat sich in die Rolle des Nesthockerhüters einschleicht (bis zur akademisch verlängerten Unreife), kann er in Interessen haben, die Frau zum Ausbrechen aus ihr zu animieren, ihr die Herrlichkeiten der emanzipierten Geldbeschafferin für den Autofetichismus der Männer vorzugaukeln. Die Macht des Staates hat nur Chancen absolut zu werden, wenn es ihr gelingt, die Mütter aus ihrer Funktion herauszudrängen - darin besteht die wahre Gemeinsamkeit aller Totalitarismen, auch der demokratisch erst sich vorbereitenden Sozialismen in ihrem unablässigen Arbeiten an der Entbindung der Frau von ihrem Primäreinfluss auf die Kinder." Der Zusammenhang von neotenie und Mitterrollenbindung in der anthropogenese und das Staatsinteressen and eren destruktion (DLA Marbach). 
palanca de las cosas una vez en la historia de la vida poniendo fin a la totalidad. Esto todavía sigue siendo posible. ${ }^{27}$

Siguiendo esta línea, comprobamos a partir de las sugerencias blumenberguianas que la mujer ha luchado por su emancipación y por sobreponerse a las leyes de la naturaleza desde tiempos inmemoriales, no sólo en los últimos tiempos, aprovechando cualquier oportunidad a la mano y convirtiéndola en una ventaja competitiva que la relevase de su dependencia del cuidado y la manutención masculina. Sin estas condicines, el paradigma evolutivo que conocemos hoy y del que procedemos y tomamos parte probablemente sería impensable. Con ello en mente, podemos concluir que la dedicación de Blumenberg a las cuestiones femeninas, aunque de manera aislada e incipiente, conduce hasta el reconocimiento de su constante esfuerzo particular por sobrevivir en igualdad de condiciones haciendo de sus aparentes desventajas las más favorables consecuencias; sin descuidar las circunstancias históricas que han envuelto su situación en cada momento. En definitiva, desde el estudio del papel de la mujer, Blumenberg no está sino reconstruyendo la historia de los débiles y devolviendo a aquellos el merecido reconocimiento de su función en el desarrollo cultural.

\section{Hacia una búsqueda del corpus filosófico blumenberguiano}

Después de lo expuesto hasta ahora ya no resulta tan extraño que el filósofo alemán hubiese compilado decenas de recortes mediáticos sobre la mujer ni que hubiera empleado tiempo en mecanografiar sus cavilaciones respecto a los mismos y a otras ideas de tal índole. Si quizá es menos común dar con documentos blumenberguianos en los que haga acopio de la situación social, política y económica de su época; estamos sin embargo acostumbrados a encontrarlo inmiscuido en la disciplina historiográfica y también -aunque todavía en menor medida- en la paleoantropológica. Es principalmente desde estas dos ramas de estudio desde las que ha llevado a cabo sus revisiones sobre la mujer con el fin de mostrar su naturaleza y lo que ha conseguido hacer de ella y ha quedado plasmado en la historia universal. Pero esta es sólo una conclusión parcial e inicial, una mera sugerencia interpretativa; pues el objetivo principal de este trabajo sólo pretendía alcanzar a dar a cono-

\footnotetext{
27 "Fast zum Schluß noch bekamen die Schwachen eine unvrohersehbare Chance, die Stärkeren zu sein, nur weil bei ihnen die Mütter das ganz und gar Unnatürliche durchgesetzt hatten, der Abwesenheit das Recht aufs Überleben zu verschaffen. (...) So hätten die Schwachen, indem sie ein einziges Mal in der Geschichte des Lebens die Schwäche zum Hebel der Dinge gemacht hatten, beinahe dem Ganzen ein Ende gesetzt. Das bleibt immer noch möglich." Die Lieblinge der Mütter in den Höhlen (DLA Marbach).
} 
cer una parte ignorada de la ríquisima bibliografía inédita blumenberguiana que abra las puertas a futuros estudios. No sólo no pretendía aventurarme a establecer una tesis sobre el papel de la mujer en la obra del filósofo, sino que lo he considerado en todo momento desaconsejable teniendo presente que, como anunciamos al principio, la muestra traida al lector no representa, probablemente, la totalidad de los textos sobre el fenómeno femenino que Blumenberg escribió. Muchos deben aguardar a ser descubiertos en el DLA y esta toma de contacto no es más que una llamada a la investigación posterior. Pero no exclusivamente dentro del marco de estudio de la filosofía blumenberguiana. Por ejemplo, sus sugerencias al respecto de la emancipación femenina y el papel antropogenético de la mujer pueden relacionarse con algunas corrientes que a través de la unión de ambas partes tratan de respaldar la posición de la mujer en la sociedad. ${ }^{28}$ En definitiva, mediante los testimonios y anotaciones de Blumenberg que han sido trabajados a lo largo de estas páginas podemos crear un acceso a una línea de trabajo inexplorada sobre el pensamiento blumenberguiano del que, como hemos comprobado, todavía quedan por acontecer algunas sorpresas y cuya relevancia está todavía por determinar.

\section{Referencias bibliográficas}

Arsuaga, J.L. y Martínez, I. (2006), La especie elegida. La larga marcha de la evolución humana, Madrid, Temas de Hoy.

Blumenberg, H. (1960), Paradigmen zu einer Metaphorologie, Bonn, Archiv für Begriffsgeschichte.

Blumenberg, H. (1966), Die Legitimität der Neuzeit, Frankfurt am Main, Suhrkamp.

Blumenberg, H. (1979), Arbeit am Mythos, Frankfurt am Main, Suhrkamp.

Blumenberg, H. (1986), Lebenszeit und Weltzeit, Frankfurt am Main, Suhrkamp.

Blumenberg, H. (1987), Das Lachen der Thrakerin. Eine Urgeschichte der

Theorie, Frankfurt am Main, Suhrkamp (Edición en castellano: (2009), La risa

de la muchacha tracia. Una protohistoria de la teoría, Valencia, Pre-textos).

Blumenberg, H. (1898), Höhlenausgänge, Frankfurt am Main, Suhrkamp.

Blumenberg, H. (2006), Beschreibung des Menschen, Frankfurt am Main, Suhrkamp.

\footnotetext{
28 Véanse las tesis de la selección femenina del profesor de psicología de la Universidad de Californa Michael Gazzaniga [1939] en su obra Human: The Science Behind What Makes Us Unique, editada por Harper Parennial en 2009 y publicada en castellano en 2010 por Paidós bajo el título ¿Qué nos hace humanos? La explicación científica de nuestra singularidad como especie o la defensa de la inteligencia femenina a través del estudio del encéfalo de la mujer de los paleoantropólogos españoles Juan Luis Arsuaga [1954] e Ignacio Martinez Mendizábal [1961] en La especie elegida. La larga marcha de la evolución humana, publicado en Temas de Hoy en 2006.
} 
Blumenberg, H. (2012), Quellen, Ströme, Eisberge, Frankfurt am Main, Suhrkamp.

Nachla $\beta$ DLA Marbach: Kein Bankkonto: Damagogie der Freiheit vom Geld; Ein Lebensthema; Konsequenz der Staatlichen Theorie des Geldes; Das KZ garantiert den Geldwert; Die Bergpredigt nach Hitler; Glaubte Hitler an die Sterne?; Zur ermordung Hitlers geboren - oder besser nicht geboren zu sein?; Politik: Schicksal, Kunst, Wissenschaft; Wer oder was man hätte wollen; Der Mann, der Hitler erschoss; Hitlers Bakterien; Die Absolute Unfreiheit des Artztes; Es wird werden, wie wenn nichts gewesen wäre; Familienväter; Definitionsversuch von Radikalismus: Abhängig von der Zweck/Mittel/Struktur; Kurzessays; Dem Feminismus zur Erinnerung; Die Zukunft der Frau; Refugium im Zoo; Säugetiere sind Lerntiere, der Mensch ist dazu noch ein Lehrtier; Zwei Theoretiker des Geldes; Das Land der Langeweile; Der Zusammenhang von neotenie und Mitterrollenbindung in der anthropogenese und das Staatsinteressen anderen destruktion; Weibliches Zuchtwahlverhalten und überleben d Schwachen als Kulturträger; Das genetische potential des terrorismus; Konvolut Materialsammlung Feminismus / Anthropologie; Von Samoa bis Gombe - Nur enttäuschungen; Alle Tierpopulationen haben Ökologien, nur die menschlichen Populationen haben Ökonomie; Im Spätfeminismus; Exogamia als erste Institution; Unbehagen XLV: Langeweile + Aktionismus. Última consulta: marzo de 2014.

Charlston, B. (2000), «Evolution and the cognitive neuroscience of awareness, consciousness and language», en Ed.in Chief, Medical Hypotheses, Oxford, Radcliffe Medical Press.

GazZAniga, M. (2010), ¿Qué nos hace humanos? La explicación científica de nuestra singularidad como especie, Barcelona, Paidós.

Heim, H. y Jochmann, W. (2000), Monologe im Führerhauptquartier 1941-1944, München, Orbis. (Edición en castellano: Trevor-Roper, Hugh (2004), Las conversaciones privadas de Hitler, 1941-1944, Barcelona, Crítica).

Ros Velasco, J., «Quellen, Ströme, Eisberge», Res Publica (Madrid), n 29, 2013, pp. 127-131.

Josefa Ros Velasco

Departamento de Historia de la Filosofía

Universidad Complutense de Madrid

josros@ucm.es 